\title{
High-Grade Atrioventricular Block Requiring Pacemaker Implantation after Cardiac Transplantation: An Unusual Complication
}

\author{
K. K. Talwar, Abhinit Gupta ${ }^{1}$, Raghav Bansal' ${ }^{1}$, Kewal Krishan ${ }^{2}$ \\ ORCID: \\ K. K. Talwar: https://orcid.org/0000-0002-7879-2680 \\ Abhinit Gupta: https://orcid.org/0000-0001-8152-5519 \\ Raghav Bansal: https://orcid.org/0000-0002-1649-8013 \\ Kewal Krishan: https://orcid.org/0000-0001-5321-0958
}

Department of Cardiology, Max Healthcare Institute Limited, Departments of ${ }^{1}$ Cardiology and ${ }^{2}$ CTVS, Max Super Speciality Hospital, New Delhi, India

\section{Abstract}

Self-limited bradyarrhythmias are commonly seen in postorthotopic heart transplantation patients. The most common cause of such bradyarrhythmias is sinus node dysfunction. Atrioventricular (AV) nodal blocks requiring pacemaker implantation remain distinctly uncommon. After ruling out reversible causes including dyselectrolytemia and drug toxicity, prolonged ischemia time of donor heart and transplant rejection should be considered as possible causes. The present case describes an uncommon occurrence of persistent high-grade AV block in postheart transplantation period ultimately requiring permanent pacemaker implantation.

Keywords: Brady-arrhythmia, cardiac transplantation, heart transplantation, high grade atrioventricular block, pacemaker implantation

\section{INTRODUCTION}

Cardiac transplantation remains the treatment of choice for end-stage heart disease. Arrhythmias are common in the orthotopic heart transplant (OHT) recipient, particularly in the early postoperative period, but are usually self-limited. Bradyarrhythmias occur in $8 \%-23 \%$ of patients after OHT depending on the case series and are probably due to sinus node dysfunction, but atrioventricular (AV) conduction disturbances requiring permanent pacemaker implantation are uncommon. ${ }^{[1]}$

\section{Case Report}

A 37-year-old patient underwent bicaval OHT for dilated cardiomyopathy. Pretransplant evaluation of donor heart was negative for any structural heart disease or conduction system disease. Baseline electrocardiogram (ECG) of donor heart was normal. The transplant procedure was uneventful with total ischemia time of $<3 \mathrm{~h}$. Immediately after the transplant, the

Submission: 06-Oct-18 Accepted: 17-Oct-18
Access this article online
Quick Response Code:

ECG was suggestive of the right bundle branch block (RBBB) with normal PR interval. The posttransplant patient was started on triple immunosuppressive therapy including tacrolimus, mycophenolate mofetil, and prednisolone as per institutional protocol. Epicardial backup pacing was kept in place as a part of the protocol. The patient was noted to have increasing pacing requirements starting from day 7 in postoperative period in view of $2: 1 \mathrm{AV}$ block [Figure 1] with intermittent complete heart block. After ruling out reversible causes (hyperkalemia and AV-blocking drugs) and procedural causes (prolonged ischemia time and perioperative injury to AV node), the evaluation for early rejection was planned. Endomyocardial biopsy and electrophysiological (EP) study

Address for correspondence: Dr. K. K. Talwar, Department of Cardiology, Max Healthcare Institute Limited, New Delhi, India. E-mail: kktalwar@hotmail.com

This is an open access journal, and articles are distributed under the terms of the Creative Commons Attribution-NonCommercial-ShareAlike 4.0 License, which allows others to remix, tweak, and build upon the work non-commercially, as long as appropriate credit is given and the new creations are licensed under the identical terms.

For reprints contact: reprints@medknow.com

How to cite this article: Talwar KK, Gupta A, Bansal R, Krishan K. High-grade atrioventricular block requiring pacemaker implantation after cardiac transplantation: An unusual complication. Int J Cardiovasc Acad 2019;5:29-31. 
were done on postoperative day 19. EP study revealed 2:1 AV conduction with prolonged HV interval $(130 \mathrm{~ms})$ suggestive of high-grade infra-Hisian AV block [Figure 2]. Histopathological examination and immunohistochemistry of the biopsy specimens were suggestive of mild acute cell-mediated rejection (the International Society for Heart and Lung Transplantation, Grade 1R) and were negative for pathologic antibody-mediated rejection [Figure 3]. Serum tacrolimus levels were found to be low, and with early cell-mediated rejection in consideration, the dose of tacrolimus and steroids was increased. However, the patient continued to have intermittent high-grade AV block. Repeat tacrolimus levels were in range. A permanent pacemaker implantation (single chamber, VVIR) was done on postoperative day 22. The patient was then discharged on immunosuppressive therapy in stable condition. On follow-up after 1 week, the patient was in sinus rhythm with RBBB [Figure 4] and pacemaker interrogation revealed minimal pacing requirement.

\section{Discussion}

Bradyarrhythmias after OHT are variably reported, but the most common cause remains sinus node dysfunction. Conduction system abnormalities leading to bradyarrhythmia are distinctly uncommon. Incomplete RBBB is the most common conduction

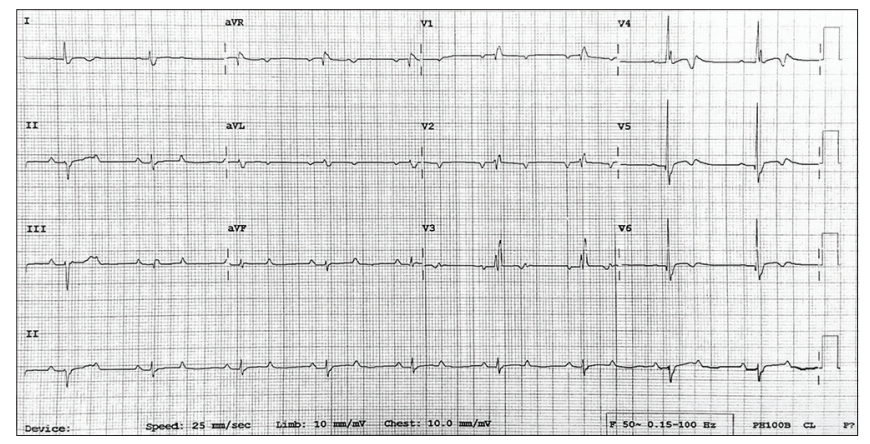

Figure 1: A 12-lead electrocardiogram done on posttransplant day 7 showing 2:1 atrioventricular block with wide QRS complex conduction (left anterior hemiblock and right bundle branch block) suggestive of infra-Hisian block

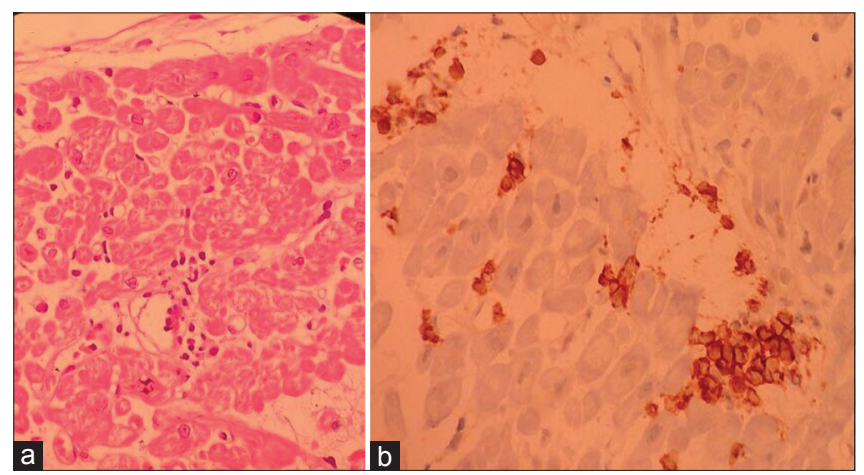

Figure 3: (a) Histopathological examination suggestive of mild acute cellular rejection (International Society for Heart and Lung Transplantation, 2004 Grade 1R) and (b) CD68 positivity on immunohistochemistry abnormality seen in up to $70 \%$ of patients following OHT. ${ }^{[1]}$ The conduction abnormality may persist and may be causally associated with prolonged donor ischemic time and episodes of rejection. ${ }^{[2]}$ However, high-grade AV block remains uncommon, particularly in the early postoperative period. According to a study of 1047 posttransplant patients, first-degree, Mobitz Type I, Mobitz Type II, and complete heart blocks occurred in $8.3 \%, 0.6 \%, 0.1 \%$, and $1.8 \%$ of patients, respectively. ${ }^{[3]}$

Whenever AV block occurs after cardiac transplantation, first reversible causes (e.g., hyperkalemia and AV-blocking drugs) should be ruled out and corrected, if any. The preoperative use of amiodarone in the recipient is an important cause of reversible posttransplant bradycardia that should not be missed. Periprocedural injury to AV node and prolonged ischemia time are also associated with postoperative AV node dysfunction and should better be avoided. Rarely, intraoperative coronary air embolism may also cause postoperative conduction disturbances. In a study by Leonelli et al., the duration of graft ischemia time and severity of rejection were the major determinants of conduction abnormality after OHT. ${ }^{[4]} \mathrm{A}$ total ischemia time $>160$ min was associated with persistence of conduction abnormality. Ice preservation of donor heart has been proposed as the likely cause of injury to conduction system. However, postoperative AV block is usually transient

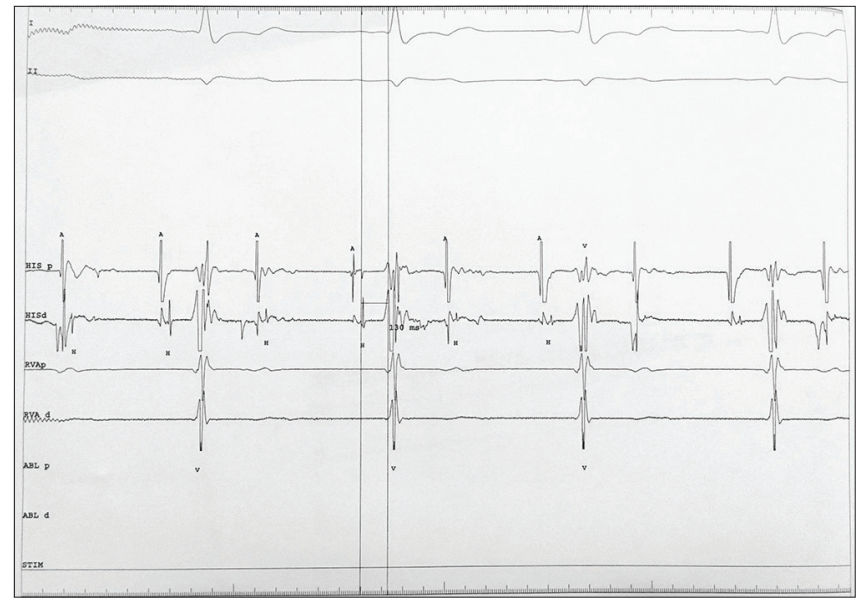

Figure 2: Electrophysiological study trace demonstrating infra-Hisian atrioventricular block (HV interval of $130 \mathrm{~ms}$ )

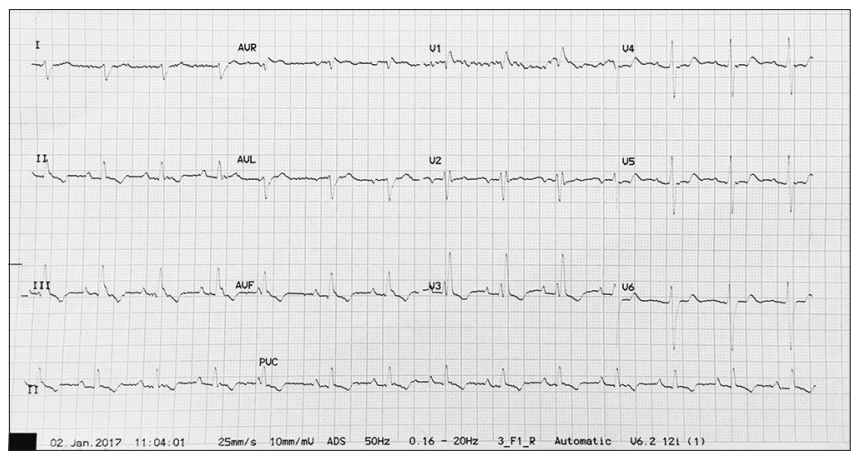

Figure 4: Normal sinus rhythm with right bundle branch block on follow-up 
and only rarely requires permanent pacemaker implantation. In a contemporary case series on 1179 transplant patients, prolonged operative time and biatrial anastomosis were identified as independent risk factors for permanent pacemaker implantation. ${ }^{[5]}$ Cardiac conduction tissue is also a specific target related to allograft rejection. The arteries to sinoatrial and $\mathrm{AV}$ tissue are frequently involved in both acute cellular reaction and chronic intimal fibrocellular rejection reaction, causing conduction disturbances. ${ }^{[6]}$ Cardiac rejection should be strongly suspected and ruled out with certainty in cases of postoperative AV blocks. Early deceleration of immunosuppressive therapy and underdosing may precipitate early rejection and should be avoided. Cardiac endomyocardial biopsy is the gold standard for diagnosing cardiac rejection and must be done whenever rejection is suspected. If the AV block persists even after treatment of rejection or EP studies are suggestive of high-risk features, implantation of permanent pacemaker may be the right approach. In the present case, EP study helped to guide the approach and tilted the balance toward advocating permanent pacemaker implantation in view of infra-Hisian block.

In a retrospective study of 114 heart transplant recipients, 14 patients $(12 \%)$ required temporary pacing and 4 patients $(3.5 \%)$ required permanent pacing. ${ }^{[7]}$ The indication for permanent pacemaker implantation was sinus node dysfunction in 3 patients $(75 \%)$ and AV block in 1 patient $(25 \%)$. They observed transplant rejection in 3 patients $(21.4 \%)$ requiring temporary pacing and in 2 patients $(50 \%)$ requiring permanent pacing. ${ }^{[7]}$ Although pacemaker implantation is performed in approximately $10 \%-15 \%$ of cardiac transplant recipients, AV block accounts for $<20 \%$ of these cases. ${ }^{[8,9]}$ Median time to pacemaker implantation is much later for AV block than for sinus node dysfunction (1511 vs. 27 days in a study by Wellmann et al.). ${ }^{[5]}$
To sum up, high-grade AV blocks are uncommon in cardiac transplant recipients (especially in early postoperative period), and if noted, acute rejection and other reversible causes should be carefully ruled before considering for permanent pacemaker implantation.

\section{Financial support and sponsorship}

Nil.

\section{Conflicts of interest}

There are no conflicts of interest.

\section{REFERENCES}

1. Hamon D, Taleski J, Vaseghi M, Shivkumar K, Boyle NG. Arrhythmias in the heart transplant patient. Arrhythm Electrophysiol Rev 2014;3:149-55.

2. Villa AE, de Marchena EJ, Myerburg RJ, Castellanos A. Comparisons of paired orthotopic cardiac transplant donor and recipient electrocardiograms. Am Heart J 1994;127:70-4.

3. Cui G, Kobashigawa J, Margarian A, Sen L. Cause of atrioventricular block in patients after heart transplantation. Transplantation 2003;76:137-42.

4. Leonelli FM, Pacifico A, Young JB. Frequency and significance of conduction defects early after orthotopic heart transplantation. Am J Cardiol 1994;73:175-9.

5. Wellmann P, Herrmann FE, Hagl C, Juchem G. A Single centre study of 1779 heart transplant patients - factors affecting pacemaker implantation. Pacing Clin Electrophysiol 2017;40:247-54.

6. Stovin PGI, Hewit S. Conduction tissue in the transplanted human heart. J Pathol 1986; 149: 183-9.

7. Bacal F, Bocchi EA, Vieira ML, Lopes N, Moreira LF, Fiorelli A, et al. Permanent and temporary pacemaker implantation after orthotopic heart transplantation. Arq Bras Cardiol 2000;74:5-12.

8. DiBiase A, Tse TM, Schnittger I, Wexler L, Stinson EB, Valantine HA, et al. Frequency and mechanism of bradycardia in cardiac transplant recipients and need for pacemakers. Am J Cardiol 1991;67:1385-9.

9. Heinz G, Kratochwill C, Hirschl M, Buxbaum P, Kreiner G, Gasic S, et al. Normal AV node function in patients with sinus node dysfunction after cardiac transplantation. J Card Surg 1993;8:417-24. 